Konrad Ott

\title{
Zur Einlagerung hochradioaktiver Reststoffe aus ethischer und politischer Sicht: Bestandsaufnahme und Ausblick
}

\begin{abstract}
This article presents some basic topics within the recent German debate on depositories for high-radioactive substances ("radwaste"). The article is organized according to two normative dimensions: security and justice. It points at different tradeoffs, for example between different research strategies, between the principles of reversibility and security, between close and remote future, and between participation and decision-making. Finally, it presents a proposal for a long-term solution.

Zusammenfassung: Dieser Aufsatz legt einige Grundlinien der neueren deutschen Debatte um die Einlagerungsstrategie von hochtoxischen radioaktiven Reststoffen dar und entwickelt hieraus einen Vorschlag hinsichtlich einer langfristigen Zielfunktion. Der Aufsatz orientiert sich an den beiden normativen Dimensionen von Sicherheit und Gerechtigkeit und analysiert einige Kompromisse wie den zwischen Reversibilität und Sicherheit, zwischen näherer und ferner Zukunft, zwischen Partizipation und Entscheidung. Zuletzt werden Thesen formuliert, die Prämissen einer diskursiven Sequenz sind, die zuletzt in eine vertretbare Zielfunktion mündet.
\end{abstract}

\section{Einleitung}

\subsection{Konfliktgeschichte}

Die Ablehnung der friedlichen Nutzung der Kernenergie stützt sich seit den 1970er Jahren im Wesentlichen auf drei Argumente: 1) das Proliferationsrisiko, das heißt der Übergang zu militärischer Nutzung, 2) das Risiko eines Reaktor-GAUs, 3) die Unsicherheit einer tatsächlich sicheren Lagerung radioaktiver Reststoffe (im Volksmund: „Atommüll“). Das erste Argument kann sich heute auf die Fälle der Staaten Nord-Korea, Pakistan und Iran berufen. Das zweite wurde durch die Unfälle von Three Mile Island, Tschernobyl und Fukushima bestätigt. Das dritte Argument kann geltend machen, dass weltweit mit wenigen Ausnahmen (Schweden, Schweiz) der endgültige Verbleib dieser extrem langlebigen und hochtoxischen Stoffe nicht geklärt ist. Alle drei Argumente haben sich demnach bewahrheitet.

Diese Gründe dürften auch die Entscheidung der Bundesregierung geleitet haben, aus der Nutzung der Kernenergie früher als geplant auszusteigen. Hatte die ,rot-grüne‘ Koalition den Ausstieg für 2023 vorgesehen und die ,schwarz-gelbe‘ Koalition ein Laufzeitverlängerungsgesetz verabschiedet, in dem die Nukleartechnik als 
„Brückentechnologie“ eingestuft wurde, schwenkte Kanzlerin Angela Merkel unter dem Eindruck von Fukushima 2011 auf die Linie um, die Jürgen Trittin als Umweltminister konzipiert hatte. Damit ist der schwerste und längste Technologiekonflikt der Bundesrepublik im Prinzip zuungunsten der Kernenergie entschieden. Es ist energiepolitisch übrigens nicht unbeachtlich, dass ca. 18 Jahre nach dem Ende der Kernenergie auch die Kohleverstromung eingestellt werden soll. Im Jahr 2040 soll Elektrizität in Deutschland ohne Atomkraft und Kohleverstromung erzeugt werden.

Nun mag man mit guten Gründen bedauern oder zornig darüber sein, dass diese atomaren Reststoffe überhaupt jemals erzeugt worden sind. Ein ,Blick zurück im Zorn` ist emotional berechtigt. Moralische Gefühle bringen diese Stoffe jedoch nicht zum Verschwinden. Die hochradioaktiven Reststoffe sind somit das Überbleibsel des „Atomzeitalters“, das ursprünglich mit so vielen Hoffnungen und Befürchtungen besetzt wurde (Bluhm 1999). Staaten sind intergenerationelle Haftungsgemeinschaften. Sie müssen auch für Fehler, Irrtümer, Schäden oder für Verbrechen der Vergangenheit einstehen. Die Reststoffe sind, so gesehen, eine technologiepolitische Altlast, die es nunmehr in einem politischen Prozess zu entsorgen gilt. Dieser Prozess steht unter normativen Vorgaben: auf demokratischem und rechtsstaatlichem Weg unter den Prinzipien größtmöglicher Sicherheit und umfassender Gerechtigkeit. Das Effizienzprinzip zählt nicht zu diesen Vorgaben, wenngleich Kosten nicht irrelevant sind.

\subsection{Framings}

In politischen Debatten werden generell framings verwendet, die einzelnen Tatsachen und Meinungen einen bestimmten Status und spezifische Bedeutung verleihen. So stellt der Atomausstieg die Problematik der Reststoffe in ein neues framing. Wurde der frühere Kampf gegen Atomkraftwerke, Wiederaufarbeitungsanlagen und geplante Endlagerstandorte von einer generellen Gegnerschaft gegen die Kernenergie zusammengehalten, können sich nunmehr auch Atomkraftgegner kaum der Einsicht verschließen, dass diese Stoffe irgendwie und irgendwo eingelagert werden müssen. Dies impliziert nicht, dass sie sich aktiv an der Problemlösung beteiligen müssen. Sie können sagen, dies sei nicht ,ihr' Problem. Sie haben auch das Recht, den politischen Prozess kritisch, ja misstrauisch, zu vorfolgen und auf dessen mögliche Defizite hinzuweisen.

Das atomkritische framing bezieht sich häufig eher retrospektiv auf die Vergangenheit des Konfliktes, das heißt auf die Proteste rund um Gorleben, auf entsprechendes ,Protestwissen', während sich ein neues framing nun prospektiv auf den eingeleiteten Atomausstieg bezieht und das Bild einer ,weißen Deutschlandkarte“ anbietet; das heißt einen neuen und seinem Anspruch nach ,besseren‘ Suchprozess initiieren 
möchte. ${ }^{1}$ Atomkraftgegnerinnen und Atomkraftgegner sind also nicht auf ein stärker retrospektives framing festgelegt. Was man sich von einer historischen Aufarbeitung des Konfliktes um die Nuklearenergie erwarten kann, ist innerhalb beider framings umstritten. Während die einen fordern, aus der Geschichte zu lernen, machen die anderen geltend, dass die Geschichtswissenschaft in der Regel kein einheitliches ,Geschichtsbild', sondern konkurrierende Narrative liefert. Das Recht des prospektiven framings liegt gewiss nicht einfach im burschikosen Gestus, nach vorne zu schauen. Wir sollten die Vergangenheit nicht einfach ,ruhen lassen', sind aber pragmatisch genötigt, prospektiv zu denken. Das anamnetische Recht des retrospektiven framing liegt darin, auf vergangene Fehler, vergangene Leistungen (wie den AkEndBericht von 2002) und ungenutzte Optionen hinzuweisen. Im Folgenden wird prospektiv argumentiert.

\subsection{Die ENTRIA-Optionen}

Mit den obigen Ausdrücken ,irgendwie“ und, irgendwo، sind zwei Dimensionen des Problems bezeichnet. Das ,irgendwie‘ bezieht sich auf die Einlagerungsoptionen, das ,irgendwo، auf die Standortfindung. Im Grunde ist die Wahl des Lagertypus dem Standortfindungsprozess vorgängig, denn falls man sich für eine dauerhafte Oberflächenlagerung ausspräche, würde sich die Standortfindung grundlegend verändern. Die trans- und interdisziplinäre Forschungsplattform ENTRIA (Entsorgungsoptionen für radioaktive Reststoffe) hat sich zwischen 2012 und 2017 unter der Wie-Frage auf drei Einlagerungsoptionen konzentriert: 1) wartungsfreies Tiefenlager ohne bzw. mit sukzessiv abnehmender Zugänglichkeit, 2) längerfristig zugängliches Tiefenlager (Stahlmann et al. 2018) und 3) langfristige Oberflächenlagerung (Ott/Budelmann 2017). Die erste Option ist der ,Klassiker‘, die zweite Option verdankt sich einer in den letzten Jahren zu verzeichnenden Aufwertung des Kriteriums der Reversibilität, die dritte Option kann als Übergangs- oder gar als Dauerlösung (sog. rolling stewardship) verstanden werden. Die beiden ersten Optionen sind mit der Wahl des Wirtsgesteins verbunden (Salz, Ton, kristallines Gestein) und verweisen somit auf die eigentliche Standortsuche. Zugänglichkeit der Stoffe ist dabei von deren Bergbarkeit im Schadensfall und von Monitoring zu unterscheiden. Das Kriterium der Reversibilität, das durch leichte Zugänglichkeit der Reststoffe erfüllt wird, ist allerdings ethisch zu reflektieren, da es zu dem Grundsatz der Sicherheit in einem Spannungsverhältnis steht, worauf zurückzukommen ist.

1 Beide framings haben zu Gorleben unterschiedliche Auffassungen: Während das retrospektive framing fordert, Gorleben aufgrund der Protestgeschichte als möglichen Standort auszuschließen, macht das prospektive framing geltend, dass sich dadurch zu Beginn des neuen Suchprozesses womöglich der Fehler des ersten Suchprozesses wiederholte, eine politische, keine fachlich begründete Entscheidung an den Anfang zu setzen. 


\subsection{Wicked Problem}

Wir haben es insgesamt mit einem sogenannten wicked problem $\mathrm{zu}$ tun, das heißt einem besonders vertrackten oder verzwickten Problem (ENTRIA-Memorandum 2014). Eine ideale risikofreie Lösung kann es nicht geben. Nach dem Maßstab dieses Ideals ist jede Lösung ,suboptimal'. Die Positionen, wie die vergleichsweise beste aller suboptimalen Lösungen aussehen könnte, sind nicht ,wahr‘ oder ,falsch', ,moralisch` oder ,unmoralisch', sondern mehr oder weniger gut vertretbar. Die bestmögliche Lösung erschließt sich nur im sachbezogenen Vergleich der Vor- und Nachteile aller (ernsthaft) infrage kommenden Optionen. Solche Vergleiche konstituieren Spielräume für vernünftige Dissense.

Ein wicked problem zieht wicked communication nach sich. Dies liegt daran, dass unisono ein Diskurs gefordert wird und das reale Sprachhandeln der Akteure zumindest teilweise strategisch-rhetorisch ist. Diskurse sind definiert als Austausch von Argumenten mit dem Ziel, idealiter einen Konsens zu finden. Strategisches Sprechhandeln dient der Beeinflussung von Kontrahenten und der Öffentlichkeit. Es wird medial inszeniert und lebt nicht zuletzt von Skandalisierungen. Aus der Teilnehmerperspektive wissen wir in der Regel, ob wir andere überzeugen oder überreden wollen. Aus der Perspektive einer Beobachtung von Redehandlungen, Dokumenten, Verlautbarungen usw. erschließt sich diese Differenz nicht ohne weiteres. Der Nachweis, hier werde etwas nur, vorgeschoben' bzw. hier zeige sich eine hidden agenda (etwa Standorte auf dem Gebiet einzelner Bundesländer auszuschließen), ist im Einzelfall schwer zu führen.

Bei der Suche nach Lösungen erweisen sich Moral und deren Reflexionsform, die Ethik, als wichtig bei der Orientierung und der Analyse, aber die Optionen lassen sich nicht allein nach moralischen Kriterien bewerten. Es muss von der ganzen Bandbreite praktischer Vernunft Gebrauch gemacht werden, das heißt es bedarf des wissenschaftlichen und technologischen Verstandes, der rechtsstaatlichen Gesinnung, der politischen Klugheit und wohl auch des nicht-persuasiven psychologischen Geschicks. Isoliert sich die Moral von den übrigen Aspekten praktischer Vernunft, schlägt sie in ihre eigene Verfallsform um, nämlich die moralisierende Besserwisserei.

\subsection{Politische Allokation eines negativen Gutes}

Ich gehe davon aus, dass die hochradioaktiven Reststoffe mehrheitlich als ,negative Güter betrachtet werden. Niemand wird in Zukunft mehr von Atomstrom profitieren, aber bestimmte Anlieger der infrage kommenden Standorte werden sich durch die Verbringung der Stoffe benachteiligt und gefährdet sehen. Bei der Verteilung negativer Güter teilen viele die Intuition, diese auf möglichst viele Schultern zu verteilen (Prinzip der Lastenteilung). Eine Lösung nach diesem Prinzip scheidet nun aber aus Sicherheitsgründen und wohl auch aus politischen Gründen aus. Was spräche dafür, 
vier oder sieben Endlagerstätten zu bauen? Wer keinen Grund anbieten kann, gibt zu, dass das negative Gut also in komprimierter Form zugeteilt werden muss. Damit ist das Prinzip der Lastenteilung jedoch nicht völlig außer Kurs gesetzt. Es könnte sich zum Kompensationsanspruch transformieren. Die auszuwählenden Standorte sind, intuitiv betrachtet, Verlierer eines „Schwarzen-Peter“-Spiels auch dann, wenn das Spiel nach fairen Regeln gespielt wird. Mit Protest, einschließlich zivilen Ungehorsams, ist daher in jedem Fall zu rechnen. Anders wäre dies nur im Falle von Freiwilligkeit, weshalb dieser Fall nicht von vornherein ausgeschlossen werden sollte (siehe unten 3.5).

Die Politik wiederum ist zur Entscheidung verurteilt. Sie kann die Entscheidungen nicht unterlassen, da sie sonst automatisch die Entscheidung trifft, die Stoffe in den jetzigen Zwischenlagern so lange wie möglich zu belassen, was aus Sicherheitsgründen keine gute Lösung ist. Eine opportunistische duck-and-cover-Strategie verschiebt die Entscheidung also nur auf andere. Entscheidungsschwäche ist ein offenes Geheimnis politischen Handelns in allen Fällen, in denen Entscheidungen Nachteile bringen. Man stelle sich beispielsweise eine ,grüne‘ Umweltministerin vor, die 2031 die Standortentscheidung ins Parlament einbringt. Einerseits soll das Parlament eine Entscheidung mit extrem langfristigen Folgen treffen, andererseits will sich die Politik durch das Kriterium der Reversibilität hiervon ein Stück weit entlasten.

Eine wichtige politische Frage ist demnach, ob eine Demokratie immer nur geschwächt und zerstritten oder vielleicht auch gestärkt aus der Lösung eines solchen wicked problems hervorgehen kann, wie immer sie inhaltlich ausfallen mag. Kann die Bürgerschaft darauf vertrauen, dass der Staat seiner Rolle als Garant einer intergenerationellen Haftungsgemeinschaft im besten Interesse der jetzigen und zukünftigen Bürgerschaft nachkommen wird? Jede Bürgerin und jeder Bürger hat einen politischen Anspruch, darauf vertrauen zu dürfen. Nun wurde im ersten Suchlauf, der sich auf den Standort Gorleben fokussierte und im alten framing der Atomwirtschaft stattfand, viel Vertrauen verspielt. Der Zustand der anderen Lagerstätten (Asse, Schacht Konrad) ist alles andere als vertrauensbildend. Allerdings hat der Prozess der letzten zehn Jahre Vertrauen auch wieder aufgebaut.

Ich setze im Folgenden voraus, dass die zu findende Lösung auf deutschem Territorium verortet werden muss, dass also ein Export dieser Stoffe nicht infrage kommt. Damit akzeptiere ich nicht nur aus Rechtstreue, sondern aus Überzeugung $\S 2$ Absatz 6 des Standortauswahlgesetzes (StandAG). Ich setze weiterhin voraus, dass die sogenannten exotischen Lösungen entweder zu riskant (Weltraum, Tiefsee) oder wissenschaftlich weit von der Einsatzreife entfernt sind (Erdkern). Ich bin skeptisch hinsichtlich der Option der Transmutation, die neue Forschungs- und Entwicklungsaktivitäten im Bereich der Nuklearindustrie erforderlich machen würde. Transmutationsforschung wäre nur in Verbindung mit langfristiger Oberflächenlagerung sinnvoll. Ich plädiere dagegen für einen klaren Schlussstrich unter die Geschichte der Nukleartechnologie. Das Geld, das für Transmutationsforschung (und meines 
Erachtens auch für Kernfusionsforschung) nötig wäre, wäre in anderen Bereichen der Klima- und Energiepolitik besser angelegt.

Die normativ-ethische Dimension wurde von ENTRIA nach zwei Dimensionen hin aufgefächert: Sicherheit und Gerechtigkeit (Walther et al. 2016). Die beiden folgenden Abschnitte orientieren sich an dieser Unterscheidung.

\title{
2 Sicherheit
}

Der unstrittige normative Grundsatz lautet, die hochradioaktiven Reststoffe seien nach bestem Wissen und Gewissen dauerhaft unschädlich zu machen, indem man sie bestmöglich bzw. möglichst sicher von der menschlichen Lebenswelt fernhält. „Unschädlich“ meint: „keinen Schaden anrichten können“, also nicht eine Umwandlung der Stoffe durch Transmutation.

\subsection{Größtmögliche Sicherheit}

Das StandAG beschreibt das Problem der Standortfindung folgendermaßen ( $§ 1$ Absatz 2):

\begin{abstract}
Mit dem Standortauswahlverfahren soll in einem partizipativen, wissenschaftsbasierten, transparenten, selbsthinterfragenden und lernenden Verfahren für die im Inland verursachten hochradioaktiven Abfälle ein Standort mit der bestmöglichen Sicherheit für eine Anlage zur Endlagerung nach § 9a Absatz 3 Satz 1 des Atomgesetzes in der Bundesrepublik Deutschland ermittelt werden. Der Standort mit der bestmöglichen Sicherheit ist der Standort, der im Zuge eines vergleichenden Verfahrens aus den in der jeweiligen Phase nach den hierfür maßgeblichen Anforderungen dieses Gesetzes geeigneten Standorten bestimmt wird und die bestmögliche Sicherheit für den dauerhaften Schutz von Mensch und Umwelt vor ionisierender Strahlung und sonstigen schädlichen Wirkungen dieser Abfälle für einen Zeitraum von einer Million Jahren gewährleistet.
\end{abstract}

Der im Gesetz genannte Zeitraum ruft natürlich allenthalben Kopfschütteln hervor, da dieser geologische Zeitraum alle wissenschaftlichen Möglichkeiten der Abschätzung übersteigt. Die letzte Eiszeit liegt gerade einmal 10.000 Jahre zurück, das heißt 1\% des genannten Zeitraumes. Die Spezies Homo sapiens sapiens dürfte nicht viel älter als 200.000 Jahre sein. Gleichwohl rechtfertigt sich dieser ,unmögliche‘ Zeitraum mit den toxischen Eigenschaften der Stoffe. Im Grunde schließt diese gesetzliche Bestimmung eine dauerhafte Oberflächenlagerung aus. Der Zeitraum erfordert eine ,geologische“ Lösung.

Sicherheit unterscheidet sich nach a) safety, das heißt nach Schutz vor der Exposition mit radioaktiver Strahlung, und nach b) security, das heißt Schutz der Stoffe vor unbefugtem Zugriff. Dass Sicherheit ein Grundwert ist, bestreitet niemand. Häufig werden qualifier wie „,best-“ oder „größtmöglich“ hinzugefügt. Die anzustrebende 
„größtmögliche“ Sicherheit ist nun nicht im Sinne des Superlativs, sondern des Elativs zu verstehen. Ähnlich wie man sagt: „Sie gehört zu den weltbesten Geigerinnen“, sagt man: „An diesem Standort herrscht größtmögliche Sicherheit“. Im Unterschied zum Superlativ, der genau eine Option als die „unübertreffliche“ auszeichnet, lässt der Elativ einen kleinen Kreis von (ungefähr) gleichrangig guten Optionen zu. Ein elativisches Verständnis von „größtmöglich“ wird dem Umstand gerecht, dass Wissenschaftlerinnen und Wissenschaftler minimale Sicherheitsdifferenzen einer Tiefenlagerung über sehr lange Zeiträume nicht zu identifizieren vermögen. Dies liegt unter anderem daran, dass jedes Tiefenlager ein Unikat ist. Es ist leichter, jemanden oder etwas in die engere Auswahl zu ziehen als ,objektiv‘ festzustellen, wer oder was das ,Beste‘ ist. Darüber muss dann häufig abgestimmt werden.

Die Suche nach größtmöglicher Sicherheit enthebt also die Politik wahrscheinlich nicht der Notwendigkeit, zwischen mehreren geeigneten Standorten entscheiden zu müssen. Diese Entscheidung kann wissenschaftlich abgestützt, aber nicht wissenschaftlich ,bewiesen' werden. Dies aber ist für die Politik eher eine schlechte Nachricht, da sie die zu treffende Entscheidung nicht dadurch rechtfertigen kann, dass die Wissenschaft den, allerbesten' Standort ermittelt habe.

\subsection{Safety Case}

Für die Abschätzung von safety sind folgende Faktoren relevant:

- Einschlusswirksamer Bergbereich,

- Eigenschaften des Wirtsgesteins (Salz, Ton, kristallines Gestein),

- Materialeigenschaften von Behältern (etwa Kupfer),

- Wärmeentwicklung, Korrosionseffekte, Austrittspfade,

- Wirksamkeit der Verbindung aus geogenen und technogenen Barrieren,

- Stabilität von Oberflächenlagern (in Hinsicht etwa auf einen Flugzeugabsturz),

- Monitoring und Bergbarkeit.

Eine interessante Frage lautet, ob die bekannten hydrologischen Nachteile kristalliner Gesteine (Granit), nämlich die Durchlässigkeit des Gesteins gegenüber Wasser, Grund genug sein sollten, dieses mögliche Wirtsgestein ohne bergtechnische Untersuchung einzelner möglicher Standorte zu verwerfen.

Hinsichtlich der Dimension von security sind die Unsicherheiten noch höher, da man die Strukturen und Ereignisse zukünftiger Gesellschaften nicht prognostizieren kann. Wer hätte 1911 Auschwitz vorhersagen können? Hinsichtlich security kann man nur mit Szenarien arbeiten, in denen virtuellen Akteuren bestimmte Motive und Anreizstrukturen zugeschrieben werden. Die Gefahr besteht, dabei unmerklich scripts aus bestimmten Filmgenres zu übernehmen. In jedem Fall haben die Einlagerungsstrategien, die Zugänglichkeit vorsehen, ein größeres security-Problem, denn sie müssen über den Verteidigungsfall nachdenken. Ein wirklicher Zivilisations- 
bruch, der etwa dem Untergang des römischen Reiches vergleichbar wäre, dürfte ein unzugängliches Tiefenlager übrigens eher sicherer machen.

Die Einstellung der interessierten Öffentlichkeit scheint dabei zwischen hohen Erwartungen und grundsätzlichem Misstrauen gegenüber Wissenschaft und Politik zu schwanken. Dieses Schwanken zählt zum wicked problem hinzu. Dagegen fällt es schwer, die Eigenarten einer interdisziplinären Forschung zu vermitteln, bei der wie in ENTRIA - Geologie, Radiologie, Bau-, Material- und Bergwerkswissenschaft, Technikfolgenabschätzung, Politikwissenschaft, Jurisprudenz und Ethik kooperieren. Interdisziplinäre Forschung kann ein hohes Maß an gesellschaftlicher Relevanz erreichen, aber bezahlt dies häufig mit einem Verlust an letzter wissenschaftlicher Sicherheit. Viele Studien zu Detailfragen der Einlagerung beruhen auf Modellen, Laborexperimenten und Simulationen. Viele der in Modellen generierten Zahlenwerte beruhen auf vorgängigen Annahmen. Die an ENTRIA beteiligten Forscherinnen und Forscher haben vielfach auf die Grenzen wissenschaftlichen Wissens hingewiesen, minimale Sicherheitsdifferenzen über extrem lange Zeiträume zu berechnen. Wissenschaftliche Ungewissheit und wissenschaftlicher Dissens vergrößern das Spektrum vertretbarer Positionen.

\subsection{Risiko und Minimax}

Bei der Frage nach größtmöglicher Sicherheit spielt das verbleibende Risiko eine wichtige Rolle. In ENTRIA waren Risikoerwägungen allgegenwärtig. Faktoren der Risikobewertung sind Eintrittswahrscheinlichkeit, Schadensausmaß und Verteilung der möglichen Schäden in Raum und Zeit. Unter der Voraussetzung der komprimierten Einlagerung der hochradioaktiven Reststoffe favorisiere ich das sogenannte MinimaxKriterium, das auffordert, eine Einlagerung so zu gestalten, dass der maximal mögliche Schaden minimiert wird. Man geht also von der „Heuristik der Furcht“ (Jonas 1979) aus und betrachtet den worst case. Im Falle der Reststoffe ist dies eine massive Kontamination der Umwelt und menschlicher Populationen mit toxischen Strahlen in einer bestimmten Höhe und in einem bestimmten Radius. Das Schadensausmaß zu minimieren, bedeutete Evakuierungen, Verbot des Anbaus von Nahrungsmitteln und der Grundwasserförderung etc. Man müsste langfristige Vorkehrungen treffen, den Radius solcher Maßnahmen, das heißt die „Verbots-Zone“ zu minimieren. Unter dem Minimax-Kriterium wäre eine Verbindung aus technogenen und geogenen Barrieren zu ermitteln, die allenfalls eine ,tröpfelnde‘ Kontamination zuließe.

Minimierungsstrategien setzen ein Wissen um den Schadenseintritt voraus. Dieses Wissen ließe sich durch Monitoringsysteme verbessern. Allerdings stellt sich bei einem langfristigen Monitoring das Problem der false positives: Ein Signal könnte einfach auf dem Veralten des Monitoringsystems beruhen, nicht auf einem realen Schadenseintritt. Deshalb wäre es womöglich besser, ein langfristiges ökologisches Monitoring der Umgebung vorzusehen. Dies wiederum setzt dauerhafte Kapazitäten 
voraus. Das Minimax-Kriterium ist damit mit der Frage nach langfristiger Governance verbunden.

\subsection{Risiko und Gefahr}

Eine Risikobetrachtung nach bestem Wissen und Gewissen bleibt auch unter dem Minimax-Kriterium einer Theorie rationaler Entscheidung verpflichtet. Niklas Luhmann (1991) hat auf die gesellschaftspolitische Differenz zwischen Risiko und Gefahr hingewiesen. Mögen die Experten das Risiko noch so genau und gründlich berechnen und es zu minimieren bestrebt sein, ändert dies nichts daran, dass betroffene Personengruppen sich gefährdet sehen. Ihnen wird eine Gefährdung auferlegt, die sie nicht freiwillig gewählt haben und der zuzustimmen sie prima facie keine Veranlassung haben. Die Wahrnehmung der Gefahr ist von der Berechnung eines Risikos verschieden. Eine optimierte Risikokalkulation verringert diese Differenz nicht, denn aus den Grenzen der Berechenbarkeit werden auf Seiten der Gefährdeten ,unberechenbare‘ Gefahren. Das Verhalten der Gefährdeten wird von Dritten als eigennütziges NIMBY-Verhalten (,Not in my backyard!“) interpretiert. Für Luhmann ist Konsens in dieser Konstellation illusorisch; eher hat die Differenz von Risiko und Gefahr aus der Perspektive eines soziologischen Beobachters zweiter Ordnung die ironische Konsequenz, dass das Sicherheitsniveau immer höher wird, während die Proteste weitergehen. Proteste wiederum haben externe Effekte auf neutrale oder affirmativ eingestellte Personen. So mag es viele Bürgerinnen und Bürger geben, die weniger Angst vor toxischer Strahlung als davor haben, den Rest ihres Lebens an einer hochpolitischen Großbaustelle verbringen zu müssen, was notorischen Ärger und Wertverlust der Immobilien bedeutet. Die Politik wird bestrebt sein, Proteste zu mäßigen und Ärger zu minimieren, und wird nur ungern mit Rechtszwang drohen. Die Dimension der Gerechtigkeit ist für Art und Ausmaß von Protest stark relevant: Empfindet eine Mehrheit der Bevölkerung das Verfahren der Standortfindung und die Wahl des Endlager-Typs als fair, erschwert dies die Mobilisierung zum Protest. Politik scheut kaum etwas mehr als ,hässliche Bilder‘ in den Medien, aber es macht einen politischen Unterschied, ob sich 1.000 oder 100.000 Menschen an Aktionen zivilen Ungehorsams beteiligen.

\subsection{Nähere und fernere Zukunft}

Bei der Langzeitverantwortung ist die Zukunft nicht homogen (Kermisch 2015; Ott/ Semper 2017). Es gibt Konflikte zwischen näherer und fernerer Zukunft. Hätte man ein bestmögliches Endlager gefunden und nach einer Phase der Noch-Zugänglichkeit wartungsfrei eingerichtet, wären die nachfolgenden Generationen bestmöglich sicher, während diese Sicherheit in sehr ferner Zukunft wieder zurückgehen könnte (allerdings nicht müsste). Die Findungsphase jedoch wird die nähere Zukunft ver- 
gleichsweise stark belasten. Standortauswahl, Herstellung von Rechtssicherheit, Bau eines Tiefenlagers mit entsprechender Infrastruktur, Transport der Reststoffe aus den Zwischenlagern, Einlagerung und allmählicher Verschluss sowie die hierbei anfallenden Kosten (vgl. unten 2.6) dürften sich bis zum Ende des Jahrhunderts hinziehen. Allein der Plan, bis $2031 \mathrm{zu}$ einer Standortentscheidung zu kommen, ist - höflich gesagt - ehrgeizig. Diese Zeitstruktur dürfte auch zu dem Problem führen, dass die Betriebsgenehmigungen der Zwischenlager und Castor-Behälter auslaufen, lange bevor eine Einlagerung möglich ist. Eine Option wäre, die Zwischenlager bautechnisch nachzurüsten. Dies würde aber bedeuten, dass die lokale Bevölkerung (etwa in Lubmin) sich von der Politik getäuscht sieht.

Prinzipiell könnte es durchaus vertretbar sein, mehr Zeit für die Suche nach einem (im definierten Sinne) bestmöglich sicheren Tiefenlager zu verwenden. Ein Argument für eine langfristige Lagerung hochradioaktiver Abfälle in Bauwerken an der Oberfläche ist der mit ihr erhoffte Zeitgewinn, das heißt der Zeitgewinn für die sich heute als langwierig erweisende Realisierung einer endgültigen Lösung. Auf dieser Linie hat schon Shrader-Frechette (1993: 215-218) argumentiert. Eines ihrer Prinzipien lautet: „Minimize scientific uncertainty by delaying the decision about permanent disposal“ (216). Eine zeitliche Verschiebung der Entscheidung über eine endgültige Einlagerung soll zu einem Zugewinn an wissenschaftlichem Wissen führen: „The main rationale for NMRS [negotiated, monitored, retrievable storage] is scientific“ (217). Allerdings führt auch die Reduktion wissenschaftlicher Unsicherheit nicht zu letzter Sicherheit hinsichtlich des Einlagerungszeitraumes. Und eine längere muss nicht unbedingt auch eine gründlichere Suche sein.

Eine längere und gründlichere Suche käme wohl der Sicherheit der späteren Zukunft zugute, dürfte aber der näheren Zukunft mehr Belastungen aufbürden. So könnte es sich in diesem Szenario als notwendig erweisen, an mehreren Standorten mit untertägigen Erkundungen nach einem Tiefenlager zu suchen und zugleich mehrere Oberflächenlager für eine Langfristzwischenlagerung zu bauen. Die Einlagerungsarbeiten vermehren sich also stark. Ob diese Strategie der Bevölkerung politisch vermittelbar ist, bleibt offen.

Die große Gefahr einer Langfristzwischenlagerung an der Oberfläche (oder in einer Art ,Tiefgarage`) liegt natürlich darin, dass die Suche nach einem Tiefenlager nicht mehr energisch verfolgt wird, weil Politiker die Konflikte scheuen (Ott/Budelmann 2017). Ergebnis wäre dann ein dauerhaftes oberflächennahes Provisorium, das weder in der safety- noch in der security-Dimension das bestmögliche Ergebnis erbringt. Vertretbar ist also auch, für eine rasche Standortfindung einzutreten. Dafür sprach sich auch das Bürgerforum (2015) aus.

Eine wichtige Frage bei der Zukunftsverantwortung, auf die Klaus-Jürgen Röhlig immer wieder hingewiesen hat, liegt darin, welche sonstigen Probleme in einer zukünftigen Welt auftreten mögen. Meine eigene Problemwahrnehmung ist nicht allzu optimistisch. Das begonnene Anthropozän könnte eine krisengeschüttelte, prekäre und ungemütliche Epoche werden (Ott 2017). Unter dieser Prämisse gewinnt der 
Gedanke an Plausibilität, zukünftige Generationen von einem wicked problem dauerhaft zu befreien, damit sie sich anderen Problemen (Klimawandel, Naturverbrauch, Migration) zuwenden können.

\subsection{Zur Kostenfrage}

Zwischen Sicherheit und Gerechtigkeit vermitteln die Kosten der Suche, des Baus und der Einlagerung. Zugewinne an Sicherheit sind kostenträchtig. Man stelle sich im Gedankenexperiment vor, dass ein bestimmter Werkstoff für die Behälter (gegenüber Kupfer) einen langfristigen Zugewinn an Sicherheit erbringen würde. Angenommen, dieser Werkstoff sei extrem teuer in der Herstellung (etwa der Preis von Gold oder Platin): Würde man sich für diesen Werkstoff entscheiden, entstünden Opportunitätskosten, da man ja auch Kupfer nehmen und mit den gesparten Kosten andere sinnvolle Dinge finanzieren könnte, etwa Anpassung an den Klimawandel, Umstellung auf organische Landwirtschaft oder die Sanierung der Deutschen Bahn. Die Opportunitätskosten von Sicherheitsgewinnen sind nicht einfach belanglos; ENTRIA hat hierzu jedoch nicht geforscht.

Als sicher gilt, dass die Rückstellungen der Betreiber von Atomkraftwerken für die Endlagerung nicht ausreichen werden. $\mathrm{Zu}$ den ,Altlasten' der Atomkraft zählen also nicht nur die Stoffe selbst, sondern die Kosten, die die Steuerzahler werden aufbringen müssen. Über die Höhe dieser zusätzlichen Kosten sind derzeit nur Spekulationen möglich. Eine genaue Berechnung der Gesamtkosten der Atomenergie, angefangen mit der staatlichen Förderung in den 1950er Jahren und endend mit den Betriebskosten eines Endlagers dürften die Rede vom „billigen Atomstrom“ als Techno-Legende entlarven.

\section{Gerechtigkeit}

Seit Aristoteles unterscheidet man mehrere Dimensionen der Gerechtigkeit: a) die politische Gerechtigkeit, die in die Lehre von den Staatsformen führt, b) die verteilende, das heißt distributive Gerechtigkeit, die in Ansprüchen des Zukommens bestimmter Güter gründet, und c) die sogenannte kommutative Gerechtigkeit im freiwilligen oder unfreiwilligen Verkehr der Bürgerinnen und Bürger untereinander, was in dem Vertragsrecht und der Straftheorie mündet. Im Anschluss an Aristoteles kann man unterschiedliche Aspekte der Gerechtigkeit bei der Endlagersuche analytisch voneinander unterscheiden, um sie aufeinander beziehen zu können. Ich unterscheide zwischen

- politischer (Demokratie)

- juridischer (Rechtsstaatlichkeit)

- prozeduraler (Beteiligung) 
- Langfristverantwortung (intertemporale Gerechtigkeit)

- distributiver (Allokation eines negativen Gutes)

- $\quad$ kompensatorischer Gerechtigkeit (Freiwilligkeit).

\subsection{Politische Gerechtigkeit}

Konsens besteht dahingehend, dass die politische Dimension der Gerechtigkeit a) die Rechtsstaatlichkeit des Verfahrens einschließlich von Klagewegen, b) die demokratische Entscheidungsfindung und c) die Partizipation der betroffenen Bevölkerung umfasst. Die Verlagerung der Entscheidungskompetenz über den Standort auf das Parlament (Legalplanung) ist prinzipiell nicht zu beanstanden, da das Parlament die höchste Input-Legitimität aufweist und ein Verwaltungsverfahren der politischen Dimension des Problems nicht gerecht wird. ${ }^{2}$

\subsection{Prozedurale Gerechtigkeit}

Das Kriterium der prozeduralen Gerechtigkeit bezieht sich auf Art und Ausmaß an Partizipation, das heißt Bürgerinnen- und Bürgerbeteiligung. Nun kann man schwächere von stärkeren Formen der Partizipation unterscheiden. Anhörungen, Stellungnahmen, Durchführung von Bürgerforen, Regionalkonferenzen usw. sind unterschiedliche Formate der Beteiligung bzw. der Mitsprache. Hinsichtlich des anzustrebenden Niveaus an Partizipation gehen die Vorstellungen allerdings weit auseinander. Während manche eine Beteiligung eher als transparenten Konsultationsprozess verstehen, fordern andere echte Veto-Rechte für Betroffene.

Der Balanceakt besteht also zwischen einem hohen Maß an Beteiligung, die gleichwohl die letzte Entscheidungskompetenz bei demokratisch legitimierten staatlichen Organen belässt. Genau dieser Balanceakt wird den Vorwürfen immer wieder neue Nahrung geben, im Grunde sei Beteiligung nur eine ,Farce‘. Wenn den Bürgerinnen und Bürgern mitgeteilt wird, dass ein Gebiet in die engste Auswahl gezogen worden sei, so erfolgt statt eines ergebnisoffenen Diskurses dann eine eher therapeutische Kommunikation, wie mit dem Befund umzugehen sei. Misslingt diese Kommunikation, entstehen ,Wutbürger‘. Das Einräumen echter Veto-Rechte wiederum könnte die Anzahl der Veto-Spieler mit der Anzahl möglicher Standorte gleichsetzen, also jede politische Entscheidung blockieren.

2 Hinsichtlich der juristischen Regulierung der Standortfindung sind die Arbeiten von Ulrich Smeddinck maßgeblich. 


\subsection{Bürgerforum}

Im Rahmen von ENTRIA wurde 2014/15 unter meiner Leitung ein sogenanntes Bürgerforum durchgeführt. Die Bürgerinnen und Bürger sprechen sich in ihrem Bürgergutachten (Bürgerforum 2015), das durch Vermittlung von Michael Müller der Kommission „Lagerung hoch radioaktiver Abfallstoffe“ vorgelegen hat, deutlich für ein Tiefenlager und eine zügige Suche nach einem solchen Lagertyp aus. Dabei regte ein Teilnehmer des Bürgerforums an, die drei ENTRIA-Lagertypen nicht als Alternativen, sondern als Module in einer temporalisierten Strategie zu verstehen. Die Oberflächenlagerung wäre dann ein mögliches Modul in einer übergreifenden, zeitlich gestreckten Entsorgungsstrategie. Interessant hierbei ist, dass eine spontane Laienintuition und das Ergebnis der Kommissionsarbeit in die gleiche Richtung weisen. Die Strategie legt somit eine gewisse Vermutung der Vernüntigkeit nahe. Offen bleibt, ob a) eine längere Oberflächenlagerung in nachgerüsteten oder neu errichteten Gebäuden vermieden werden kann, und b) wie die Zugänglichkeit eines auszuwählenden Tiefenlagers allmählich zu reduzieren wäre.

\subsection{Reversibilität und ihre Paradoxien}

Es führt kein Weg an der Einsicht vorbei, dass angesichts der verschiedenen Handlungsoptionen ein Paternalismus der Gegenwart über die Zukunft unvermeidlich ist. „Paternalistisch“ meint hier: Es ist keine Entscheidung möglich, die nicht stellvertretend für zukünftige Generationen getroffen wird. Die durch die Zeit selbst gegebene Macht der Gegenwart über die Zukunft erweist sich als etwas, dessen man sich nicht entschlagen kann. Dürfen die Lebenden eine bestimmte Lösung in die Tat umsetzen, die ihnen nach bestem Wissen und Gewissen sowie nach ausführlicher Deliberation als die ,beste، erscheint, selbst wenn sie dadurch zukünftige Generationen vor vollendete Tatsachen stellen und ihnen das Risiko eines Fehlschlags aufbürden?

Freilich kann man versuchen, sich dem Paternalismus zu entziehen. Beispielhaft hierfür ist die folgende Aussage: „Nachfolgende Generationen müssen unsere Entscheidungen revidieren können, gleichzeitig dürfen wir sie nicht mit dem Zwang zu handeln belasten“ (Kotting-Uhl 2016). Zum einen wird die Forderung nach Reversibilität beibehalten und zum anderen soll die Entscheidung der gegenwärtigen Generation Handlungsdruck auf zukünftige Generationen vermeiden. Reversibilität wird bestmöglich durch die Zugänglichkeit des Endlagers gewährleistet. Allerdings muss ein zugängliches Endlager im Berg oder an der Oberfläche kontinuierlich gewartet und überwacht werden, was klarerweise Handlungszwänge für zukünftige Generationen mit sich bringt. Zugängliche Endlager zu vernachlässigen, ist auch in Zukunft unverantwortlich.

Die geforderte Reversibilität der Entscheidungen impliziert aber auch, in näherer oder fernerer Zukunft ein zugängliches in ein unzugängliches Lager überführen zu 
dürfen, obwohl diese Überführung dann praktisch irreversibel ist. Oder soll Zugänglichkeit irreversibel sein? Da es technisch immer möglich sein dürfte, die Zugänglichkeit einzuschränken, bräuchte es einen starken moralischen Grund, der die gesamte Kette zukünftiger Generationen verpflichtete, die Zugänglichkeit zu gewährleisten. Einen solchen Grund kenne ich nicht.

Rein formell betrachtet würde die Verbringung der Stoffe in ein unzugängliches Tiefenlager die Handlungsfreiheit zukünftiger Generationen zwar einschränken, doch ob dies mit der substantiellen Freiheit der Zukünftigen, ihr Leben individuell und kollektiv nach eigenem Gutdünken zu gestalten, weniger gut vereinbar wäre als ein rolling stewardship, bei der eine dauerhafte Bewachung zugänglicher Einlagerungsstätten vorgesehen wäre, steht keineswegs fest. Warum sollten fernere Generationen die Belastung einer Dauerüberwachung einem unzugänglichen Tiefenlager vorziehen? Es besteht keine moralische Verpflichtung zukünftiger Generationen, die Zugänglichkeit immer weiter zu gewährleisten. Dies gilt für die nähere und die fernere Zukunft gleichermaßen. Wenn dies gilt, so könnte auch bereits um das Jahr 2100 herum das Endlager unzugänglich gemacht werden.

\subsection{Freiwilligkeit}

Es wurde eingangs dargelegt, dass die Allokation eines negativen Gutes mit einem „Schwarzer-Peter“-Spiel verglichen werden kann, an dessen Ende ein Verlierer feststeht. Dieser kann, wenn das Spiel fair gespielt wurde, mit seinem Pech hadern, aber sich nicht über eine Ungerechtigkeit beschweren. Dieses Gleichnis trifft dann nicht mehr auf das Auswahlverfahren zu, wenn eine Region sich bei vorausgesetzter Geeignetheit in der Sicherheitsdimension bereitfände, freiwillig zur Standortgemeinde zu werden. Den Freiwilligen geschieht ja kein Unrecht. Abstriche an der Sicherheit wären nicht zulässig, aber Freiwilligkeit könnte die Rolle eines tie breakers spielen. Für die Politik wäre Freiwilligkeit eine ungeheure Entlastung, da sie dann nur ein Angebot aufgreifen müsste. Freiwilligkeit bedeutet weniger Protest, weniger Ärger, weniger Verzögerungen, weniger Transaktionskosten, weniger Skandalisierung usw.

Wir können zwischen ,starker' und ,schwacher' Freiwilligkeit unterscheiden (Ott/ Riemann 2018). Starke Freiwilligkeit bedeutet, ein Opfer für ein Kollektiv zu bringen, dem man sich zugehörig fühlt. Dieses Sich-Opfern wurde in der Geschichte häufig heroisierend überhöht. Eine säkulare, post-heroische Gesellschaft fordert von ihren Mitgliedern kein Opfer. Schwache Freiwilligkeit ist die Bereitwilligkeit, eine Last gegen eine angemessene Entschädigung (Kompensation) anzunehmen. Solche Kompensationslösungen verdeutlichen natürlich den Paternalismus der Gegenwart, da sich die Bereitwilligkeit zukünftiger Generationen nicht abfragen lässt. Während die starke Freiwilligkeit, ethisch betrachtet, ein übergebührliches (,supererogatorisches“) Handeln ist, ist auch die schwache Freiwilligkeit aus Sicht der gesamten Bürgerschaft immerhin noch ein verdienstvolles (,meritorisches“) Handeln. Gegen frei- 
willige Kompensationslösungen lässt sich immer der moralisch gemeinte Vorwurf der moral corruption vorbringen, das heißt einer an Bestechlichkeit grenzenden oder ihr gleichkommenden Käuflichkeit. Aber Kompensationsverhandlungen sind, wenn sie freiwillig erfolgen, vom Handlungstyp her keine pflichtwidrige Annahme von Geldzuwendungen. Schwache Freiwilligkeit schillert somit je nach Sichtweise zwischen meritorischem und verräterisch-korruptem Handeln.

Entlastet man schwache Freiwilligkeit von moralischen Vorwürfen, wären Art und Höhe der Kompensation zwischen Staat und Region verhandelbar. Das ökonomische Problem, sowohl Über- als auch Unterkompensation zu vermeiden, sollte bei diesen Verhandlungen nicht allzu streng genommen werden. Sollte eine Region unterhalb des Median-Durchschnittswohlstands liegen, könnte man sogar überproportional kompensieren, um im Endeffekt die Gleichwertigkeit der Lebensverhältnisse zu befördern.

Hinsichtlich der Art der Kompensation bräuchte man sich nicht an den Wohlstandmustern der frühen Bundesrepublik zu orientieren (Hallenbad, Autobahnzubringer, Sportanlagen usw.). Möglich wäre auch, ,postmoderne‘ Kompensationsangebote vorzulegen (frühere Verrentung, Kindertagesstätten, ökologische Landwirtschaft, ÖPNV usw.). Vom Modell einer ,Entwicklung‘ peripherer Räume könnte man sich guten Gewissens verabschieden. Ein Problem könnte hingegen darin liegen, dass neue und attraktive, ja verlockende Angebote den Kreis der Standortgegnerinnen und -gegner spalten könnten. Bei strenger Voraussetzung der Sicherheitsaspekte, gelebter Freiwilligkeit der Akteure und attraktiven Angeboten könnte der zu erwartende Vorwurf an Gewicht verlieren, der Erfolg von Kompensations-,,Deals“ gründe in der Ungleichheit der Lebensverhältnisse und die Lasten würden am Ende den Armen aufgebürdet.

\section{Fazit}

Wie immer man das Problem dreht und wendet; die Gesellschaft ist zu einer Lösung, die Politik ist zu einer Entscheidung verurteilt. Bei der Entscheidungsvorbereitung und -findung hat sich ein komplexer Argumentationsraum konstituiert, in dem es viele gute und plausible, aber nicht den eindeutig ausschlaggebenden Super-Grund gibt, der bestimmt, wie zu handeln sei. Die beste aller möglichen Lösungen lässt sich auch nicht aus Prinzipien der Sicherheit und Gerechtigkeit deduzieren. Man kann also keine Beweise führen, sondern nur abwägen und beurteilen. Was kann unter dieser Voraussetzung die philosophische Ethik als Ergebnisse ihrer Arbeit und als Beiträge zur politischen Debatte um die Einlagerung vorweisen? Folgende Punkte sind mir wichtig: 
1. Hochradioaktive Stoffe und ähnlich toxische Substanzen sollten nie wieder erzeugt werden.

2. Deren Einlagerung muss konzentriert erfolgen; dilute-and-disperse-Lösungen sind abzulehnen.

3. Größtmögliche Sicherheit ist im Sinne eines Elativs zu verstehen; die Wissenschaft wird womöglich keine eindeutige first-best-solution ermitteln können.

4. Ein safety case muss wissenschaftsbasiert sein.

5. Das Minimax-Kriterium ist (unter den in Abschnitt 2 genannten Voraussetzungen) zur Risikobeurteilung sinnvoll.

6. Zwischen Zugänglichkeit und Sicherheit bestehen Konflikte. Reversibilität ist kein oberster Wert. Zugänglichkeit sollte nur eine Übergangslösung sein.

7. Längerfristige Oberflächenlagerung an alten oder neuen Standorten wäre der Preis einer gründlichen untertägigen Erkundung mehrerer geologischer Standorte. Dies ist eine aufwändige Lösung mit dem möglichen Nachteil, dass ein langfristiges Provisorium entstehen könnte.

8. Die zu wählende Einlagerungsstrategie sollte die zukünftige Welt insgesamt sicherer und konfliktärmer machen.

9. Ein Paternalismus der Gegenwart über die Zukunft ist unvermeidlich.

10. Es gibt einen Trade-Off zwischen naher und ferner Zukunft. Geringfügige Vorteile für die ferne Zukunft müssen mit erheblichen Belastungen für die nahe Zukunft abgewogen werden.

11. Jeder Generation ist es erlaubt, ein zugänglich gebautes Lager zu verschließen. Es gibt keine dauerhafte Pflicht zur Offenhaltung.

12. Prozedurale Gerechtigkeit kann nicht bis zu Veto-Rechten der Anlieger führen.

13. Der Aufbau der Governance-Struktur kann im Wesentlichen als abgeschlossen gelten.

14. Die Letztverantwortung für Entscheidungen sollte beim Parlament liegen.

15. Die Bereitwilligkeit, die Last gegen angemessene Kompensation zu übernehmen, wäre meritorisches Handeln.

16. Eine argumentativ vertretbare Zielfunktion ist demnach: ein praktisch unzugängliches Tiefenlager mit einem Gefüge aus geogenen und technogenen Barrieren, das (elativisch) größtmögliche Sicherheit unter einem Minimax-Kriterium bietet, in Verbindung mit Vorkehrungen zur Bergbarkeit und mit einem ökologischen Oberflächenmonitoring an einem Standort, dessen Bürgerinnen und Bürger die Einlagerung der radioaktiven Reststoffe gegen angemessene Kompensation bereitwillig auf sich genommen haben oder den das Parlament nach ausführlicher Entscheidungsvorbereitung und Beratung für den „bestgeeignetsten“ ansieht. 


\section{Literatur}

AkEnd (2002). Auswahlverfahren Endlagerstandorte: Empfehlungen des AkEnd - Arbeitskreis Auswahlverfahren Endlagerstandorte [Abschlussbericht]. Unter Mitarbeit von D. Appel, B. Baltes, V. Bräuer, W. Brewitz, K. Duphorn, R. Gömmel et al. Köln [https://www.bundestag.de/ endlager-archiv/blob/281906/c1fb3860506631de51b9f1f689b7664c/kmat_01_akend-data. pdf; 05.03.2019].

Bluhm, H. (1999). Das Atomzeitalter: Varianten einer Epochenbestimmung. In: K. Fischer. Hrsg. Neustart des Weltlaufs. Frankfurt/Main: Suhrkamp, 203-224.

Bürgerforum (2015). Bürgergutachten des Bürgerforums „Wohin mit unserem Atommüll?“ [https:// www.bundestag.de/resource/blob/365600/a762d9d615164690cb2957db510cb605/ kmat_20-data.pdf; 05.03.2019].

Jonas, H. (1979). Das Prinzip Verantwortung: Versuch einer Ethik für die technologische Zivilisation. Frankfurt/Main: Suhrkamp.

Luhmann, N. (1991). Soziologie des Risiko. Berlin: de Gruyter.

Kermisch, C. (2015). Specifying the Concept of Future Generations for Addressing Issues Related to High-Level Radioactive Waste. Science \& Engineering Ethics 22(6), 1797-1811 [DOI: 10.1007/ s11948-015-9741-2].

Kotting-Uhl, S. (2016). Endlagersuche mit Chance auf Akzeptanz [https://gruenekarlsruhe.de/ endlagersuche-mit-chance-auf-akzeptanz/; 05.03.2019].

Ott, K. (2017). Verantwortung im Anthropozän und Konzepte von Nachhaltigkeit. In: M. Luber, M. Patenge und R. Beck. Hrsg. Schöpfung bewahren: Theologie und Kirche als Impulsgeber für eine nachhaltige Entwicklung (Weltkirche und Mission 7). Regensburg: Pustet, 64-103.

Ott, K. und H. Budelmann (2017). Oder vielleicht doch nicht unter der Erde: Überlegungen zur Rolle der Oberflächenlagerung in einer Entsorgungsstrategie. In: D. Köhnke, M. Reichardt und F. Semper. Hrsg. Zwischenlagerung hoch radioaktiver Abfälle: Randbedingungen und Lösungsansätze zu den aktuellen Herausforderungen. Wiesbaden: Springer, 11-27.

Ott, K. und F. Semper (2017). Nicht von meiner Welt: Zukunftsverantwortung bei der Endlagerung von radioaktiven Reststoffen. GAIA. Ecological Perspectives for Science and Society 26(2), 100-102 [DOI: 10.14512/gaia.26.2.9].

Ott, K. und M. Riemann (2018). „Volenti non fiat injuria“: Freiwilligkeit und Bereitwilligkeit bei der Übernahme von Standortverantwortung. In: K. Ott und U. Smeddinck. Hrsg. Umwelt, Gerechtigkeit, Freiwilligkeit - insbesondere bei der Realisierung eines Endlagers. Berlin: Berliner Wissenschafts-Verlag, 41-57.

Röhlig, K.-J. et al. (2014). ENTRIA: Memorandum zur Entsorgung hochradioaktiver Reststoffe [https:// www.entria.de/fileadmin/entria/Dokumente/ENTRIA_Memorandum_140430.pdf; 05.03.2019].

Shrader-Frechette, K. S. (1993). Burying Uncertainty: Risk and the Case against Geological Disposal of Nuclear Waste. Berkeley: University of California Press.

Smeddinck, U. (2016). Umgang mit Ungewissheit bei der Realisierung eines Endlagers. In: H. Hill und U. Schliesky. Hrsg. Management von Ungewissheiten und Nichtwissen. Baden-Baden: Nomos, 147-183.

Stahlmann, J. et al. (2018). Normalszenarien und Monitoringkonzepte für Tiefenlager mit der Option Rückholung: Generische Tiefenlagermodelle mit Option zur Rückholung der radioaktiven Reststoffe. ENTRIA-Arbeitsbericht 15 [https://www.entria.de/fileadmin/entria/Dokumente/ Arbeitsberichte/ENTRIA_Arbeitsbericht-15_Rueckholung_und_Monitoring.pdf; 05.03.2019]. StandAG (2017). Gesetz zur Suche und Auswahl eines Standortes für ein Endlager für hochradioaktive Abfälle [https://www.gesetze-im-internet.de/standag_2017/BJNR107410017.html; 05.03.2019]. 
Walther et al. (2016). Entsorgungsoptionen für hochradioaktive Abfälle: Die Schaffung interdisziplinärer Bewertungsgrundlagen in ENTRIA. Vortrag an der Jahrestagung des Fachverbands für Strahlenschutz, Heringsdorf Usedom, 28.09.2016. 